\title{
Erinnerungen an Edgardo Giovannini (1909-2004) und die Freiburger Zeit
}

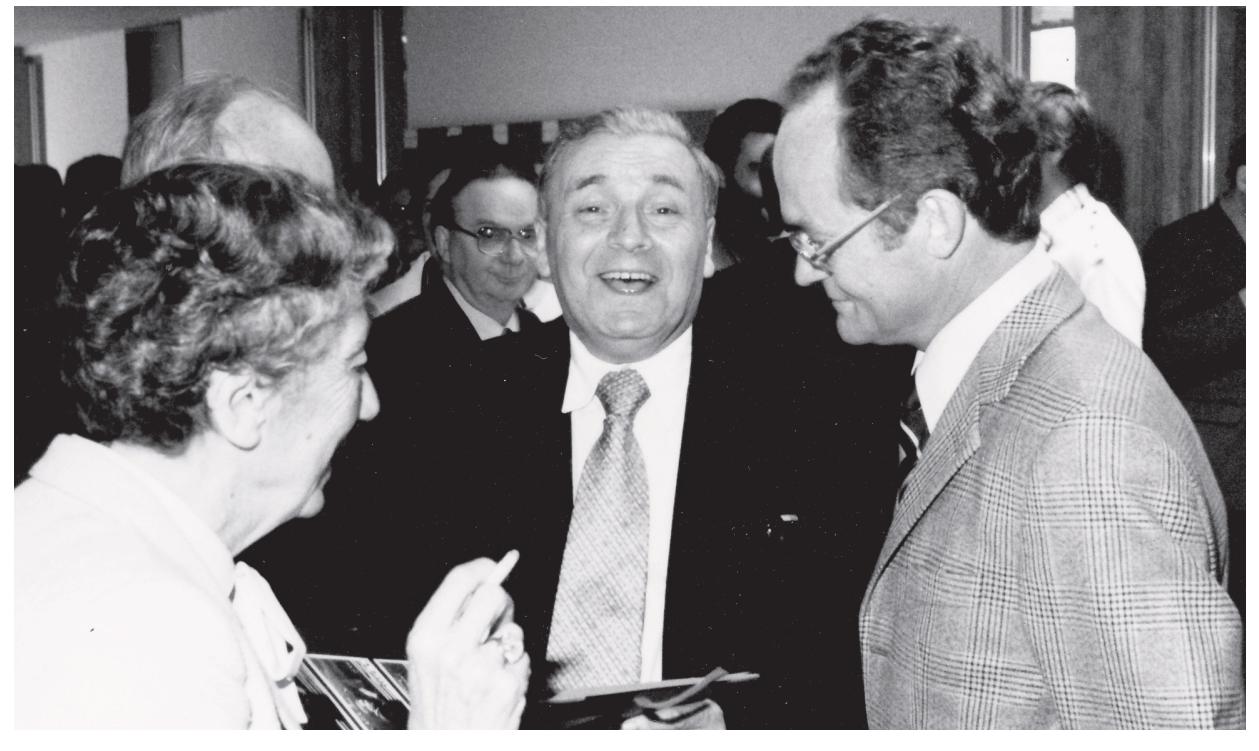

Am Donnerstag, den 22. Januar dieses Jahres, fand in Fribourg in der Eglise Sainte-Thérèse die Trauerfeier für Edgardo Giovannini statt, der Anfang der Woche, am 19. Januar 2004, nach einem langen, stets kämpferisch erfüllten Leben in seinem 95. Lebensjahr gestorben war. Edgardo Giovannini war zweifellos die am stärksten Einfluss nehmende Persönlichkeit auf die Freiburger Universitätschemie der 60er und 70er Jahre des vergangenen Jahrhunderts. Anteil daran hatte sein zweimaliges Amten als Dekan der Faculté des Sciences (195253 und 1958-59) sowie die darauf folgende Zeit als Rektor (1966-68) und Vizerektor (1968-70) der Universität Freiburg. In ihm und mit ihm vollzog sich in Freiburg der Übergang von der Chemie des auslaufenden 19. Jh. und frühen 20. Jh., die geprägt war von Augustin Bistrzycki (1862-1934), der 1896 mit der Etablierung der naturwissenschaftlichen Fakultät an die Universität Freiburg berufen worden war und hier als Professor für analytische und technische Chemie bis zu seinem Rücktritt 1932 tätig war, sowie von Henri de Diesbach (18801970) - bekannt durch seine Entdeckung der Phthalocyanine - der von 1920-1955 als Professor für organische Chemie wirkte, und schliesslich, als Schüler de Dies- bachs und Nachfolger Bistrzyckis, Louis Chardonnens (1898-1976), der von 19341969 einen Lehrstuhl für analytische und physikalische Chemie innehatte. In diese Zeit hinein fällt das Chemiestudium von Edgardo Giovannini an der Universität Freiburg, das er 1928 begann und mit einer Doktorarbeit unter der Leitung Bistrzyckis mit dem Titel «Über Kondensationsprodukte von Acetophenonderivaten mit Methylolverbindungen» und nach kurzen Studienaufenthalten an der ETH Zürich und Universität Zürich 1935 abschloss.

Edgardo Giovannini wurde am 24. Juni 1909 in Lugano als ältestes Kind des Bankbeamten Arnoldo Giovannini und der Giuseppina Primavesi, deren Eltern ein Kolonialwarengeschäft in Lugano besassen, geboren. Sieben weitere Geschwister folgten. Seine klassische Schulbildung erhielt er am Gymnasium in Lodi in der Lombardei. In das Jahr seines Studienabschlusses fiel seine Heirat mit der Klavierlehrerin Edith Wirz, die selbst einer Familie, in der Vater und Mutter Lehrer waren, entstammte. Wenn man gemeinhin von Kindersegen spricht, so wurde er exemplarisch für das Ehepaar Giovannini, denn im Laufe der Jahre wurden ihnen elf Kinder geboren, von denen zwei inzwischen verstorben sind.
Prof. Edgardo Giovannini an der Verabschiedungsfeier anlässlich seines 70 . Geburtstages im Salle d'Etude des Chemiegebäudes 1979. Links im Vordergrund: Anna Chylewska, Sekreträrin für Helvetica Chimica Acta; die hohe Stirn hinter ihr gehört zum damaligen Rektor der Universität Freiburg, Prof. Bernhard Schnyder; Mitte Hintergrund (mit Brille): Prof. Emil Adolf Siegrist; rechts im Vordergrund: Prof. Christian Caron, damaliger Dekan der Faculté des Sciences der Universität Freiburg

Um der neuen Familie existenzielle Sicherheit zu geben, nahm Edgardo Giovannini nach Abschluss seiner Studien noch 1935 die Stelle eines Assistenten des Kantonschemikers der Urkantone in Brunnen an. Im Jahre 1938 folgte der Wechsel auf eine Stelle der Verwaltung der Armee in Wimmis. In der Armee selbst erlangte er den Rang eines Obersten der Gebirgsdivision. Als ihm 1943 von der Universität Freiburg ein Extraordinariat für organische und theoretische Chemie angeboten wurde, war seine weitere Laufbahn als Hochschullehrer in Fribourg vorgezeichnet. Fünf Jahre später (1948) erfolgte seine Ernennung zum Ordinarius und schliesslich 1955, als Nachfolger Henri de Diesbach's, seine Einsetzung als Direktor des Institut de chimie organique, ein Amt, das er bis zu seinem Altersrücktritt 1979 einnahm.

Nach der Übernahme der Leitung des Instituts für organische Chemie durch Edgardo Giovannini wurde zum WS 1955/56 Oskar Klement (1912-1986), ein ausgesprochener «Valence-Bond»-Theoretiker, als Professor für physikalische Chemie nach Fribourg berufen. Im Jahr 1977 trat er aus gesundheitlichen Gründen von seinem Amt zurück. Noch vor dem Rücktritt von Louis Chardonnens konnte in der organi- 
schen Chemie eine Assistenzprofessur neu besetzt werden. Auf Vorschlag Giovanninis wählte die Fakultät aus der jungen Garde der nachrückenden ETH-Hochschulforscher und -lehrer Rolf Scheffold (19321994), der von 1968 bis zu seinem Ruf im Jahr 1972 auf den frei gewordenen Lehrstuhl von Rudolf Signer am Institut für organische Chemie der Universität Bern als Assistenzprofessor in Fribourg die Disziplinen Stereochemie und synthetische Chemie vertrat. In die Zeit von Rolf Scheffold fiel auch die Ernennung von PD Emil Adolf Siegrist, der in der Ciba, Basel, als Forschungschemiker die sogenannte Anilsynthese von Stilbenen (in der Schweiz deshalb auch Siegrist-Reaktion genannt) zur Produktionsreife gebracht hatte, zum Titularprofessor (WS 1970/71-WS 1990/91) mit dem Lehrgebiet Chemie der Farbstoffe. Rolf Scheffold setze sich im Einverständnis mit Prof. Giovannini auch dafür ein, dass die HMO-Theorie in Fribourg gelehrt wurde. Reinhart Keese, Oberassistent am Laboratorium für organische Chemie der ETH (ab 1974 Oberassistent und dann ab 1976 Professor am Institut für organische Chemie der Universität Bern), nahm dafür einen Lehrauftrag zum WS 1968/69 an, den er bis 1981 ausübte. Zu diesem Zeitpunkt war Edwin Haselbach aus der Schule Edgar Heilbronners schon auf den Klement'schen Lehrstuhl für physikalische Chemie nachgerückt und zum Direktor des entsprechenden Instituts ernannt worden, so dass HMO-Theorie «in house» gelehrt werden konnte. Edwin Haselbach trat nach dem SS 2001 von seinem Amt zurück.

Kurz nach der Berufung von Rolf Scheffold trat Louis Chardonnens altershalber zurück, und es galt den Lehrstuhl für anorganische Chemie zukunftweisend mit einem Nachwuchsforscher zu besetzen. Die Wahl fiel auf einen Studiumskollegen von Rolf Scheffold an der ETH Zürich, Alex von Zelewsky, der zum WS 1969/70 als damals jüngster Ordinarius der Chemie in der Schweiz nach Fribourg kam und auch die Leitung des Instituts für anorganische Chemie übernahm. Auf seinen Vorschlag hin wurde zur gleichen Zeit Franzpeter Emmenegger als Assistenzprofessor, insbesondere für Festkörperchemie, an das Institut für anorganische Chemie berufen. Sein Altersrücktritt erfolgte zum SS 2001. Der Verantwortungs- und Generationenwechsel in der Chemie an der mathematisch-naturwissenschaftlichen Fakultät der Universität Freiburg hatte also Ende der 60er und Anfang der 70er Jahre unter der Ägide Edgardo Giovanninis stattgefunden, und auch die Lücke, die durch den Wechsel von Rolf Scheffold an die Universität Bern entstanden war, wurde sofort wieder geschlossen, indem der Schreibende zum SS 1973 als Assistenzprofessor für organische Chemie nach Fribourg berufen wurde.
Was fand der Schreibende vor? Erst einmal ein in der letzten Phase des Entstehens sich befindendes, wunderbares neues Chemiegebäude, das mit Geldern des Bundes, des Kantons und der Ciba errichtet wurde, das grosszügige, geräumige und harmonisch konzipierte Laboratorien bot und einen hervorragend ausgerüsteten grossen und kleinen Hörsaal besass. Dazu kam noch ein bald einmal auch für Sitzungen gern genutzter Salle d'Étude gegenüber den Hörsälen. Die Rückwand des grossen Hörsaals schmückte ein im Cibachrome-Verfahren hergestelltes Bild von Hans Erni, welches das Periodische System der Elemente künstlerisch deutete. Die Baukommission für das neue Chemiegebäude, das vom Lausanner Architekten Arthur Lozeron entworfen worden war, der nach seinem Tod durch seinen jungen Freiburger Kompagnon Serge Charrière ersetzt wurde, präsidierte Staatsrat Aebischer, und Alex von Zelewsky vertrat darin die Belange der Chemie. Der Schreibende erinnert sich noch gern an die Detailsitzungen unter dem jungen $\mathrm{Ar}$ chitekten Charrière mit allen Chemieprofessoren, an denen letzte Änderungen bei Raumausstattungen beschlossen wurden. Zum Herbst 1974 konnte das neue Gebäude im Pérolles endlich bezogen werden. Alles war grossartig und ausgewogen und atmete eine wunderbare Zukunft des Lehrens und Forschens. Es versteht sich, dass ein solcher Neubau einer langjährigen politischen und planerischen Vorarbeit bedarf, die zweifellos von Edgardo Giovannini als Rektor und Vizerektor in der Zeit von 1966-1970 geleistet wurde. Dazu kamen alte, aufgebrauchte und $\mathrm{zu}$ kleine, noch aus den Anfängen unter Bistrzycki stammende Gebäudekomplexe, günstige wissenschaftspolitische Entscheidungen auf Bundesebene, es sei in diesem Zusammenhang an den Krebser-Bericht und das Hochschulförderungsgesetz unter Bundesrat Tschudi erinnert, und das entschiedene Mitwirken der frisch tätigen Chemieprofessoren, allen voran Alex von Zelewsky als massgeblicher Anorganiker.

Nun ja, das andere, was der Schreibende vorfand, war eine durch Generationenfriktion hervorgerufene eisige, auf das administrativ Notwendigste sich beschränkende Arbeitsatmosphäre zwischen den Instituten für organische und anorganische Chemie, deren eigentliche Ursachen er nie erfuhr. Aber das Kämpferische, Furiose und, wenn es sein musste, Ab- und Ausgrenzende gehörte zum Charakter von Edgardo Giovannini. Er konnte geübt väterlich, aber auch tyrannisch sein. Im ersten Fall konnte man viel bei ihm und mit ihm erreichen, vor allem wenn er erkannte, dass sein Vertrauen nicht ausgenutzt wurde. Im zweiten Fall, vor allem wenn sein Patronat angezweifelt wurde, war nicht gut Kirschen essen mit ihm, man war kurzweg durchge- fallen, ein Zustand, aus dem man sich kaum noch befreien konnte. In seinem Institut selbst herrschte eine sehr gute Stimmung und der Schreibende konnte bald einmal eine stattliche Anzahl deutsch- und französischsprachiger Mitarbeiter um sich versammeln. Das Lehrangebot in organischer Chemie wurde durch die Vergabe von Lehraufträgen zum WS 1974/75 an Dr. Henri Ramuz von der Pharmaforschung der F. Hoffmann-La Roche, Basel, für die Chemie der Heterocyclen und an Dr. Theodor Völker, Leiter des damaligen Instituts für Polymerforschung der Lonza im Pérolles, für die Chemie der Polymeren, ergänzt und bereichert. Henri Ramuz wurde zum SS 1979 zum Titularprofessor ernannt und trat altershalber zum WS 1997/98 aus dem Lehrbetrieb aus. Mit der Beförderung des Schreibenden zum Extraordinarius (WS 1976/77) war die Möglichkeit gegeben, eine im Entwicklungsplan des Instituts vorgesehene zweite Stelle eines Assistenzprofessors am Institut für organische Chemie endlich zu besetzen. Aber darum musste vom Institutschef gekämpft werden. Die sich über mehrere Semester erstreckenden N.N. bei gewissen organischen Lehrveranstaltungen in den Vorlesungsverzeichnissen jener Jahre legen beredt Zeugnis davon ab. Endlich war es soweit. Herr Giovannini rief mich in sein Eckbüro, um mir mitzuteilen, dass wir voranmachen könnten und dass er einen möglicherweise interessanten ehemaligen Vierer-Lizentiaten von Fribourg, Sohn eines früheren Kollegen für Schweizer Geschichte, der an der ETH Zürich bei Duilio Arigoni promoviert habe und der erst vor kurzem nach einem Forschungsaufenthalt in den Vereinigten Staaten auf eine Oberassistentenstelle am Laboratorium für organische Chemie der ETH zurückgekehrt sei, für die zu besetzende Assistenzprofessur im Auge habe. Es würde sich um Andrea Vasella handeln. Aber er möchte keine Entscheidungen ohne mein ausdrückliches Einverständnis treffen. Ich solle doch Andrea Vasella einmal beschnuppern, um ihn näher kennen zu lernen. Also machte sich der Schreibende auf nach Zürich zu einem Gespräch mit dem Ins-Auge-Gefassten. Das Gespräch verlief respekt- und vertrauensvoll, und die diskutierten Arbeiten über 1,3-dipolare Cycloadditionen mit Nitronen, abgeleitet aus geschützten Monosacchariden, waren neu und viel versprechend. Die Wahl von Andrea Vasella zum Assistenzprofessor für organische Chemie durch die mathematisch-naturwissenschaftliche $\mathrm{Fa}$ kultät verlief nicht ganz im Sinne der organischen Chemie und schon gar nicht des Kandidaten, denn die Fakultät machte entgegen den Zusicherungen von Prof. Giovannini zur Auflage, dass der Gewählte eine Habilitationsschrift nachzureichen habe. Andrea Vasella trat seine Professur zum WS 1977/78 nicht ohne Groll an, zumal auch 
die Freigabe der Gelder des ihm zugesicherten Einrichtungskredites auf sich warten liess. Die geforderte Habilitationsschrift war bald einmal unter Dach und Fach gebracht und die Gelder freigegeben. Die schwierigen Startumstände waren überwunden, aber nicht vergessen. Zum WS 1981/82 nahm Andrea Vasella den Ruf auf ein Extraordinariat für organische Chemie der Universität Zürich an.

Nachzutragen bleibt, dass es Mitte der 70er Jahre auch eine Verstärkung der physikalischen Chemie gegeben hatte. Nach vorsichtigen, tastenden Gesprächen mit den Kollegen hatte sich Oskar Klement entschieden, Werner Hug, der seinen Forschungsaufenthalt in der Gruppe von Ignacio Tinoco in Berkeley beenden wollte, als Oberassistent an sein Institut zu holen. Werner Hug kam 1975 mit klar gefassten Plänen für den Bau eines ROA-Spektrometers (ROA = Raman Optische Aktivität) an das Institut für physikalische Chemie. Es folgten drei arbeitsintensive Jahre, um mit Geldern des Nationalfonds und einer eingeschworenen kleinen Gruppe das Spektrometer zu konstruieren. Und im Herbst 1978 war es soweit, die ersten ROA-Spektren konnten in einzigartiger Qualität aufgenommen und ein Jahr später der RuzickaPreis der ETH Zürich für Nachwuchsforscher von Werner Hug in Empfang genommen werden. Die ROA-Forschungen wurden jäh beendet, als am Freitag, den 13 März 1981, ein verheerendes Feuer, ausgebrochen im Institut für organische Chemie, grosse Teile des Ostflügels des Chemiegebäudes verwüstete. Durch Hitze- und Raucheinwirkung wurde auch das im obersten Stock des Ostflügels sich befindende ROA-Spektrometer irreparabel geschädigt.

Als Edgardo Giovannini nach dem SS 1979 altershalber zurücktrat und die Verantwortung für das Institut für organische Chemie auf den Schreibenden überging, konnte er sehr zufrieden sein (siehe Photo von der Verabschiedungsfeier im Salle d'Étude des Chemiegebäudes; für weitere Photos und Würdigungen Edgardo Giovanninis zu seinem 60. und 70. Geburtstag, siehe Chimia 1969, 23, 237 und 1979, 33, 225). Er übergab seinem Nachfolger ein exzellent funktionierendes Institut mit moderner analytischer und spektroskopischer Infrastruktur und hoch motivierten Mitarbeitern. Die Zusammenarbeit mit den anderen beiden chemischen Instituten, die auch gewachsen waren, klappte reibungslos.

Betrachtet man das wissenschaftliche Werk von Edgardo Giovannini, so muss man kritisch anmerken, dass es in vielen Teilen fragmentarisch geblieben ist, was allerdings angesichts der Bürde, die sich aufzuladen er stets bereit war, nicht verwundert. Neben den erwähnten Dekanatsund Rektoratszeiten war er von 1962-64 Präsident der Schweizerischen Chemischen

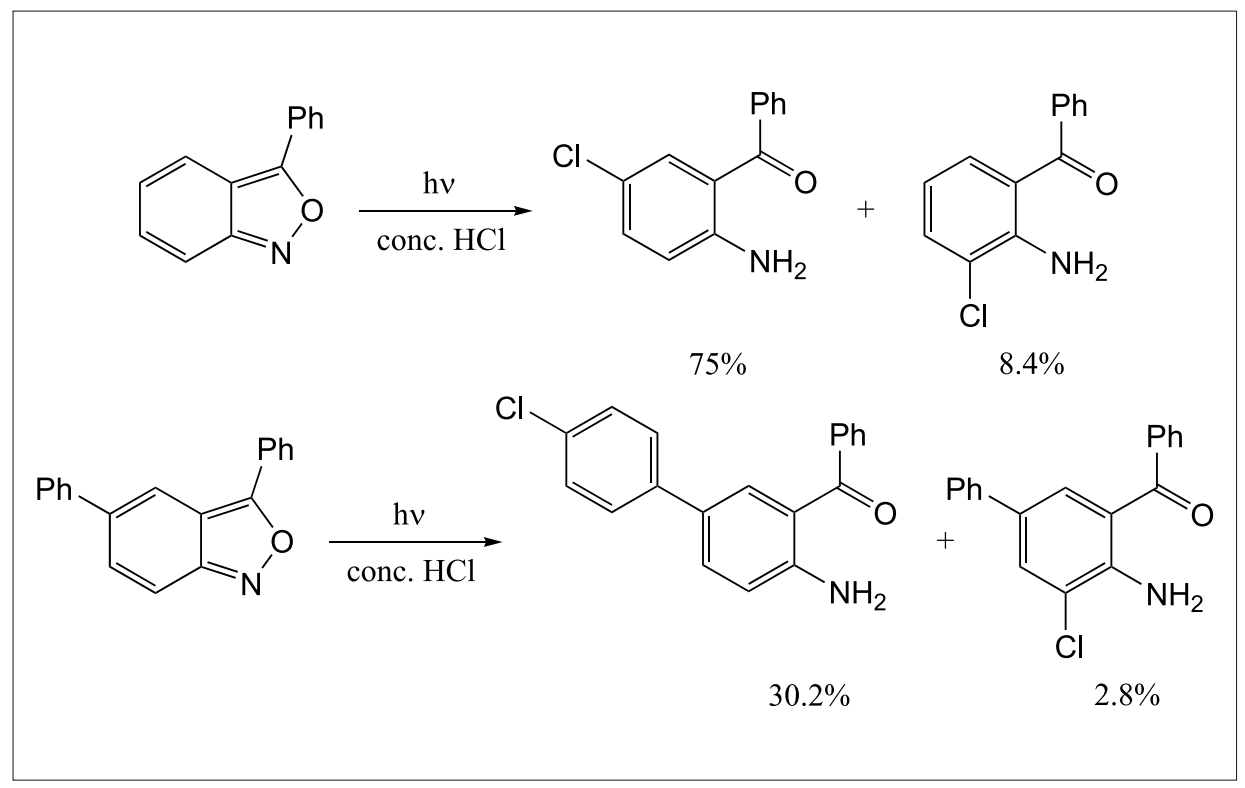

Schema 1

Gesellschaft, trat 1967 in das Redaktionskomitee von Helvetica Chimica Acta (HCA) ein, wurde 1970 Vizepräsident und dann 1972, als Nachfolger von Emil Cherbuliez, Präsident des Redaktionskomitees. $\mathrm{Zu}$ dieser Zeit amtete er auch als Forschungsrat der Sektion 2 des Nationalfonds. Die Herausgabe der HCA forderte ihm ein enormes Arbeitspensum ab. Im Jahr 1983 trat er nach diversen Querelen vom Amt des Präsidenten des Redaktionskomitees zurück. Nach einem Reorganisationsjahr in Basel unter der Leitung des Schreibenden und mit einem unvergleichlichen Einsatz des jungen vollamtlichen Redaktors, Dr. Volkan M. Kisakürek, ging die Präsidentschaft des Redaktionskomitees an Edgar Heilbronner in Basel über. Die Schweizerische Chemische Gesellschaft verlieh Edgardo Giovannini 1983 die Ehrenmitgliedschaft der Gesellschaft in Anerkennung seiner geleisteten Dienste für die Herausgabe von $H C A$. Die Società chimica italiana hatte ihm schon 21 Jahre zuvor die Ehrenmitgliedschaft verliehen, und 1978 war es die Universität Lausanne, die ihm den Doktortitel ehrenhalber verlieh.

Nun zu seinen wichtigsten veröffentlichten wissenschaftlichen Arbeiten, die, abgesehen von einigen Vorausmitteilungen im Bulletin der Freiburger Naturforschenden Gesellschaft, alle in $H C A$ erschienen sind. Den Auftakt bilden vier Arbeiten (HCA 1948, 31, 1361, 1375, 1381 und 1392), die auf der Dissertation seines ersten Doktoranden, Plato Portmann (von 1958WS 1990/91 Professor für Biochemie an der Universität Freiburg) beruhen und sich im breitem Rahmen mit Isatin- und Oxindol-Chemie beschäftigen, vor allem im Hinblick auf den Abbau von Aminosäuren und anderer Aminabkömmlinge. Diese Ar- beiten finden ihre Fortsetzung in den Publikationen der Jahre 1953-1963 (HCA 1953, 36,$708 ; \mathbf{1 9 5 7}, 40,249$ und 1553; 1958, 41, $113 ; \mathbf{1 9 6 3}, 46,1326)$, in denen von der Isatin-Chemie übergeleitet wird zur Chemie der Indoline und Indole. Ganz anders geartet sind Arbeiten dieser Jahre, die sich mit der Birch-Reduktion von Indan beschäftigen, die, wie erstmals gezeigt wird, ausschliesslich 4,7-Dihydroindan in hoher Reinheit liefert (HCA 1958, 41, 933), das dann der Epoxidierung mit Persäuren unterworfen wird, wobei ganz bevorzugt die zentrale Doppelbindung reagiert ( $H C A$ 1959, 42, 1142), so dass vergleichende $\mathrm{Ki}$ netiken der Epoxidierung von anderen olefinischen Bindungen angestellt werden konnten. Interessant ist eine vergleichsweise einfache Synthese der Indan-4-yl-essigsäure, über die berichtet wird ( $H C A$ 1966, 49, 561). Eine spätere Arbeit beschäftigt sich mit der Synthese des angulären Di(cyclobuteno)benzols (HCA 1977, $60,1452)$.

Besonders stolz war Edgardo Giovannini auf die Entdeckung der photochemischen Ringöffnung von Anthranilen in starken Säuren, die unabhängig erfolgte von gleichen Beobachtungen, die in der Zürcher Gruppe um Hans Schmid an der Universität bei der Photolyse von $1 H$ - und $2 H$-Indazolen, Anthranilen und Benzoxazolen im sauren Milieu gemacht wurden. Die Arbeiten erschienen 1971 und 1978 (HCA 1971, 54, $2111 ; \mathbf{1 9 7 8}, 62,185$ und 198). Die entsprechenden protonierten Verbindungen werden im angeregten Zustand heterolytisch zu Nitreniumionen (bzw. Oxeniumionen bei Benzoxazolen) gespalten, die dann am aromatischen Kern Nucleophile einfangen (vgl. Schema 1). Das Interessante ist, dass die Freiburger Gruppe zeigen konnte, dass 


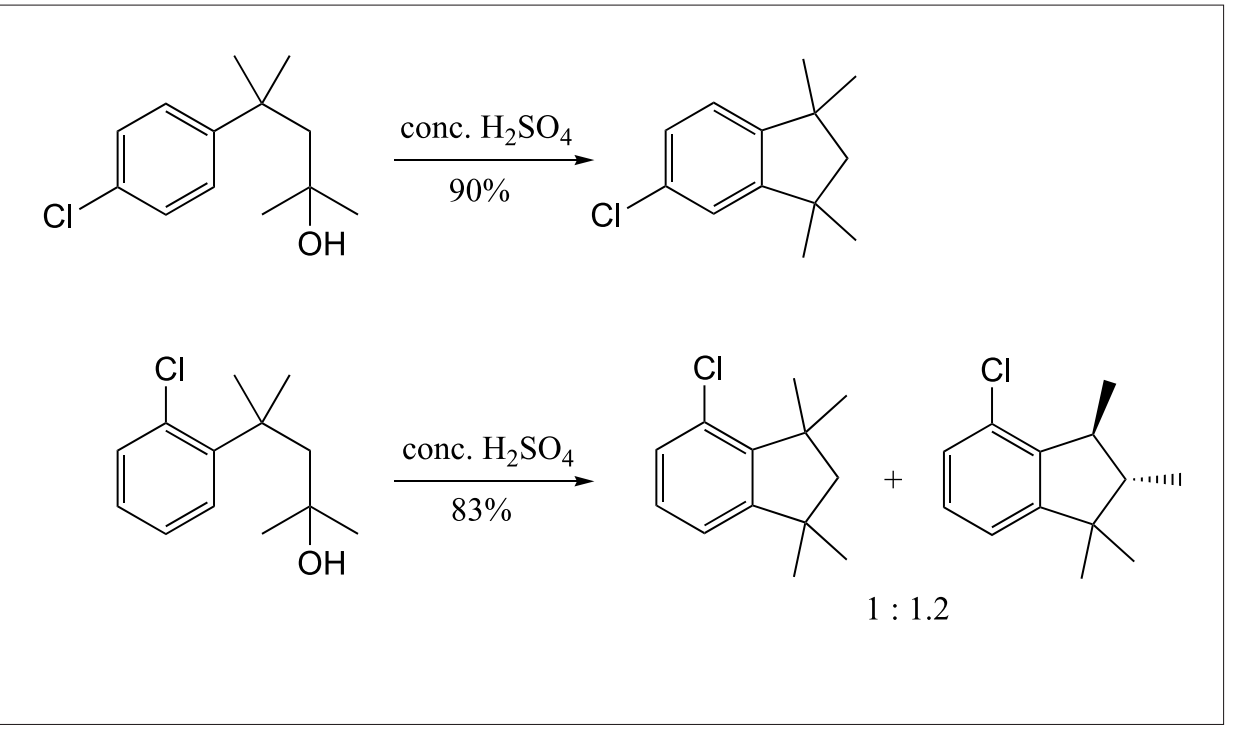

Schema 2

5-Phenyl-substituierte Anthranile bei der Photolyse Nucleophile zur Hauptsache in $p$-Stellung des zweiten Phenylkerns aufnehmen.

Die ganze Begeisterung der letzten 15 Jahre seiner Forschertätigkeit galt aber der Untersuchung der Cyclialkylierungsreaktionen von Arylalkanolen in Schwefelsäure, bei denen unvorhersehbare Alkyl- und Hydridverschiebungen vor dem Ringschluss eintreten konnten. Cyclialkylierungsreaktionen, begleitet von Alkylwanderungen, unter Friedel-Crafts-Bedingungen waren zwar bekannt und intensiv von Royston M. Roberts untersucht worden, aber die Vielgestaltigkeit der Umlagerungen der Arylalkanole in conc. Schwefelsäure oder Schwefelsäuremonohydrat, von denen das Schema nur einen kleinen Einblick vermitteln kann, war immer wieder überraschend (Schema 2).

Leider erschien über diese in der Endphase der Hochzeit der Olah'schen Carbokationen-Chemie entstandenen Arbeiten nur eine kurze Mitteilung (HCA 1973, 56, 1775). Andere wichtige Interessen und Ereignisse nahmen Professor Giovannini nach dem Rücktritt vom Amt des Präsidenten des Redaktionskomitees von $H C A$ in Anspruch. Aber die alte Kämpfernatur brach sich in dem Zweiundneunzigjährigen noch einmal Bahn, und er reichte 2001 fünf Arbeiten über Cyclialkylierungen zur $\mathrm{Pu}-$ blikation bei $H C A$, seinem chemischen Journal, ein. Sie erschienen 2002 (HCA 2002, 85, 1827, 1841, 1850, 2083 und 2089), zu spät, um die organisch-chemische Forschung der Gegenwart noch wirklich zu erreichen, aber doch Zeugnis ablegend von den Möglichkeiten mechanistischer Erforschung multipler Carbeniumionen-Umlagerungen, die den chemischen Instituten der Universität Freiburg in den 70er Jahren des letzten Jahrhunderts zu Gebote standen, nicht zuletzt dank des stets kämpferischen Einsatzes von Edgardo Giovannini für die Modernisierung der Chemie in Freiburg, die immer auch seine Chemie war.

Der Schreibende trat nach dem WS 1980/81 von seinem Freiburger Amt zurück, um in der F. Hoffmann-Roche AG, Basel, in den Zentralen Forschungseinheiten (ZFE) die Leitung der chemischen und mikrobiologischen Forschung zu übernehmen. Er leitete noch interimistisch das Institut für organische Chemie in Freiburg, in dem es galt, die durch das Feuer am 13.3.1981 verursachten Schäden zu beseitigen. Zwei Menschen halfen ihm dabei in ganz besonderer Weise, nämlich der damalige Oberassistent Reinhard Neier, seit dem WS 1991/92 Professor für organische Chemie an der Universität Neuenburg, und Direktionsinspektor William Meystre von der Suisse Mobiliar in Bern, der den Schadensfall in verständnisvoller und grosszügiger Weise regelte, so dass das Giovannini'sche Institut dem Nachfolger, Albert Gossauer, in wieder gutem Zustand und mit einem finanziellen Polster für Nachbesserungen zum WS 1982/83 übergeben werden konnte.

Zürich, am 13. November 2004

Hans-Jürgen Hansen 